\title{
DNA-Assembled Plasmonic Waveguides for Nanoscale Light Propagation to a Fluorescent Nanodiamond
}

Gür, Fatih N.; McPolin, Cillian P.T.; Raza, Søren; Mayer, Martin; Roth, Diane J.; Steiner, Anja Maria; Löffler, Markus; Fery, Andreas; Brongersma, Mark L.; Zayats, Anatoly V.

Total number of authors:

12

Published in:

Nano Letters

Link to article, DOI:

10.1021/acs.nanolett.8b03524

Publication date:

2018

Document Version

Peer reviewed version

Link back to DTU Orbit

Citation $(A P A)$ :

Gür, F. N., McPolin, C. P. T., Raza, S., Mayer, M., Roth, D. J., Steiner, A. M., Löffler, M., Fery, A., Brongersma, M. L., Zayats, A. V., König, T. A. F., \& Schmidt, T. L. (2018). DNA-Assembled Plasmonic Waveguides for Nanoscale Light Propagation to a Fluorescent Nanodiamond. Nano Letters, 18(11), 7323-7329.

https://doi.org/10.1021/acs.nanolett.8b03524

\section{General rights}

Copyright and moral rights for the publications made accessible in the public portal are retained by the authors and/or other copyright owners and it is a condition of accessing publications that users recognise and abide by the legal requirements associated with these rights.

- Users may download and print one copy of any publication from the public portal for the purpose of private study or research.

- You may not further distribute the material or use it for any profit-making activity or commercial gain

- You may freely distribute the URL identifying the publication in the public portal 


\section{DNA-assembled Plasmonic Waveguides for}

\section{Nanoscale Light Propagation to a Fluorescent}

\section{Nanodiamond}

Fatih N. Gür ${ }^{1}$, Cillian P. T. McPolin ${ }^{2}$, Søren Raza ${ }^{3, \dagger}$, Martin Mayer ${ }^{1,4}$, Diane J. Roth ${ }^{2}$, Anja

Maria Steiner ${ }^{1,4}$, Markus Löffler ${ }^{1}$, Andreas Fery ${ }^{1,4,5}$, Mark L. Brongersma ${ }^{3}$, Anatoly V. Zayats ${ }^{2}$,

Tobias A. F. König ${ }^{1,4, *} \&$ Thorsten L. Schmidt ${ }^{1,6, *}$

\section{AUTHOR ADDRESS}

${ }^{1}$ Center for Advancing Electronics Dresden (cfaed), Technische Universität Dresden, 01062

Dresden, Germany

${ }^{2}$ Department of Physics, King's College London, Strand, London, WC2R 2LS, UK

${ }^{3}$ Geballe Laboratory for Advanced Materials, Stanford University, Stanford, CA 94305-4045, USA

${ }^{4}$ Leibniz-Institut für Polymerforschung Dresden e.V., Institute of Physical Chemistry and Polymer Physics, Hohe Str. 6, 01069 Dresden, Germany

${ }^{5}$ Department of Physical Chemistry of Polymeric Materials, Technische Universität Dresden, Hohe Str. 6, 01069 Dresden, Germany 
${ }^{6} B$ CUBE - Center for Molecular Bioengineering, Technische Universität Dresden, 01062 Dresden, Germany.

\section{TABLE OF CONTENT GRAPHICS}

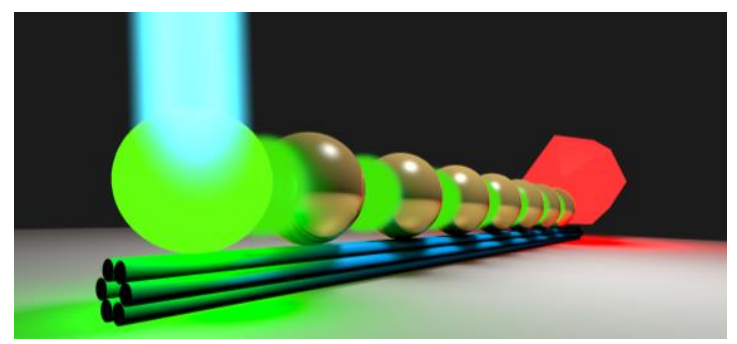




\begin{abstract}
Plasmonic waveguides consisting of metal nanoparticle chains can localize and guide light well below the diffraction limit, but high propagation losses due to lithography-limited large interparticle spacing have impeded practical applications. Here, we demonstrate that DNAorigami-based self-assembly of monocrystalline gold nanoparticles allows the interparticle spacing to be decreased to $\sim 2 \mathrm{~nm}$, thus reducing propagation losses to $0.8 \mathrm{~dB}$ per $50 \mathrm{~nm}$ at a deep subwavelength confinement of $62 \mathrm{~nm}(\sim \lambda / 10)$. We characterize the individual waveguides with nanometer-scale resolution by electron energy-loss spectroscopy. Light propagation towards a fluorescent nanodiamond is directly visualized by cathodoluminescence imaging spectroscopy on a single-device level, therefore realizing nanoscale light manipulation and energy conversion. Simulations suggest that longitudinal plasmon modes arising from the narrow gaps are responsible for the efficient waveguiding. With this scalable DNA origami approach, micrometer-long propagation lengths could be achieved, enabling applications in information technology, sensing and quantum optics.
\end{abstract}

\title{
KEYWORDS
}

DNA nanotechnology, plasmonics, nanoparticle chain waveguide, electron energy loss spectroscopy, cathodoluminescence imaging spectroscopy, fluorescent nanodiamonds 
Plasmonic structures are currently considered in numerous strategies for the miniaturization of optical and optoelectronic components. ${ }^{1-4}$ The field of plasmonics exploits the interaction of light with nanoscale metallic structures to confine, guide and manipulate light on scales below the diffraction limit, ${ }^{5,6}$ thereby greatly benefiting applications such as quantum photonics or short-range optical communications. ${ }^{1-4}$ In particular, deep-subwavelength plasmonic waveguides consisting of closely-spaced metal nanoparticle chains have been proposed for nanoscale optical applications $^{7}$ and realized mainly by electron beam lithography. ${ }^{6,8-10}$ Lithography is, however, a slow, expensive, and non-scalable method. Moreover, the metal nanoparticle shape, crucial in this case, cannot be precisely controlled by the lift-off procedures and the interparticle gaps are usually limited to $10 \mathrm{~nm}$ or more. These lithography-related fabrication issues have led to nanoparticle chains with large propagation losses, impeding their application as deepsubwavelength waveguides.

In contrast to lithography, chemical synthesis of colloidal nanoparticles offers a more precise control over the metal particle crystallinity, shape, and size. Such colloidal nanoparticles have been self-assembled into optically large $(\sim \lambda / 2)$ plasmonic waveguides with micrometer-long propagation lengths using lithographically prepared trenches. ${ }^{11,12}$ Here, we leverage on the programmability and scalability of DNA nanotechnology ${ }^{13-23}$ to push the confinement below the diffraction limit $(\sim \lambda / 10)$ while retaining a long propagation length. The robust DNA origami $\operatorname{method}^{24,25}$ is used to pattern a large variety of inorganic nanoparticles or other functional elements including proteins, or small molecules, with nanometer precision on two or three dimensions. ${ }^{15,24}$ In this manner, metal nanoparticle plasmonic devices with a static ${ }^{18}$ or reconfigurable ${ }^{26}$ circular dichroism and plasmonic hotspots for the sensing of fluorescence molecules ${ }^{17,23}$ have been realized. Self-assembled gold nanoparticle chain waveguides on DNA 
origami have also been reported, however, without demonstrating optical mode propagation ${ }^{19,20}$ or only showing a minimal energy transfer over $35-50 \mathrm{~nm}$ by far-field optical measurement. ${ }^{21}$ Thus far, the energy loss in experimentally realized ${ }^{6,8-10,19,21}$ and theoretically simulated ${ }^{8,27}$ gold nanoparticle chain waveguides reduces the incident field by around 1-2 orders of magnitude (or by $10-20 \mathrm{~dB}$ ) after a propagation length of only $50 \mathrm{~nm}$, which hinders practical applications that require propagation lengths of hundreds of nanometers or micrometers. While lower losses can be achieved with much larger waveguides, such as plasmonic discs, ${ }^{10,28}$ dielectric waveguides, ${ }^{29,30}$ metal stripes with sizes of several $100 \mathrm{~nm},{ }^{31}$ or multi-layer ribbons of nanoparticles, ${ }^{11,12}$ nanoparticle chain waveguides enable much smaller device footprints, scalable fabrication by self-assembly, and realization of more complex geometries such as corners or splitters. For this, the large propagation losses need to be mitigated.

Here, we experimentally demonstrate that losses in nanometer-sized self-assembled single particle line chain waveguides with an unprecedented mode size of only $62 \mathrm{~nm}$ can be drastically reduced from more than $10 \mathrm{~dB}^{21}$ to $0.8 \mathrm{~dB}$ per $50 \mathrm{~nm}$ by reducing interparticle gaps to few nanometers. We used these waveguides for local excitation of a fluorescent nanodiamond. The proposed approach will be useful for efficient building of nano-optical circuits for quantum technology and sensing applications, among others.

For this, we self-assembled plasmonic chain waveguides from oligonucleotide-functionalized gold nanoparticles (AuNPs, Figure 1a, d) ${ }^{20}$ binding to six-helix bundle (6-HB) DNA origami nanotubes (Figure 1b, e), which display binding sites for eight AuNPs, and studied their optical properties at a single-device level with nanometer resolution. The propagation losses can be mitigated through increased plasmonic coupling between the nanoparticles,${ }^{32}$ making it crucial to minimize the interparticle spacing. ${ }^{11,12,18}$ We therefore use AuNPs with a diameter roughly equal 
to the distance between binding sites $(42.2 \mathrm{~nm}$; Figure 1a, b). As the hydrodynamic size of the oligonucleotide-functionalized AuNPs is larger than the distance between the binding sites on the 6-HB, the particles minimize their free energy during the drying process, ${ }^{33}$ thereby minimizing the interparticle spacing, which occasionally results in slightly deformed zigzag patterns (Figure 1g). This mechanical compression of the protective oligonucleotide layer around the AuNPs allowed us to reduce the gap sizes from around $7 \mathrm{~nm}$, for mechanically uncompressed functionalized AuNPs, ${ }^{34}$ to $1.5 \pm 0.9 \mathrm{~nm}$ (Figure S1). Such a small interparticle spacing cannot be routinely achieved by conventional lithography methods, but are crucial for efficient long-range waveguiding. ${ }^{11,12,18}$ We fabricated both complete waveguides, with eight AuNPs, and incomplete waveguides where we omit the binding sites for two particles in the center of the waveguide, serving as a negative control (Figure 1c, g).

To characterize the waveguides, which contain feature sizes of only few nanometers, at a single-device level, we employ two electron-microscopy-based near-field spectroscopy techniques, where a sub-nanometer-sized focused electron beam rather than a diffraction-limited light beam serves as the energy source. First, electron energy loss spectroscopy (EELS) ${ }^{35,36}$ is used to reveal the different surface plasmon (SP) modes that are supported by the waveguides. To detect energy transport along the waveguide via the SP modes, at a single-device level, we place a fluorescent nanodiamond $(\mathrm{FND})^{37}$ at one end of the waveguide. After excitation of the waveguide by the electron beam in the cathodoluminescence (CL) imaging spectroscopy setup $^{28,38}$, energy propagates towards the FND which emits photons into the far-field. 

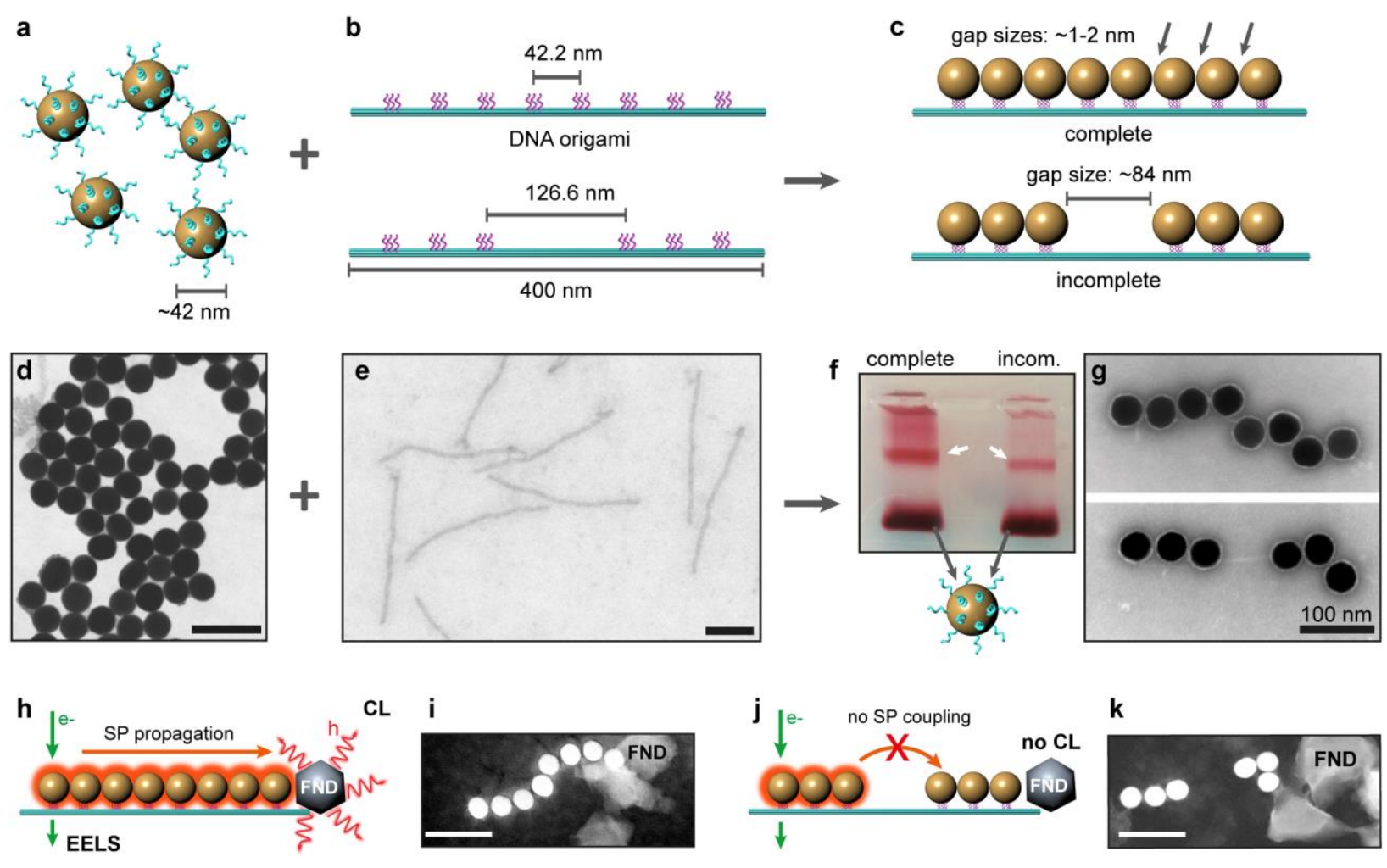

Figure 1. Self-assembly of plasmonic waveguides. Schematic representation (a-c), electron micrographs (d, e, g, i, k) and agarose gel electrophoresis (f) results of complete and incomplete waveguides. (h) Schematic representation of the energy transfer to a fluorescent nanodiamond (FND) and (i) experimental realization. Surface plasmon (SP) modes are excited by an electron beam and the loss of energy is detected by electron energy loss spectroscopy (EELS). The SPs propagating towards a fluorescent nanodiamond excite a fluorescence signal that is detected by cathodoluminescence (CL) imaging spectroscopy. (j) Schematic of an incomplete waveguide and (k) its experimental realization. SPs do not couple efficiently across the large gap, resulting in the absence of a signal from the FND when the isolated sections of the incomplete waveguides are illuminated. Scale bars: $100 \mathrm{~nm}$. 
EELS provides the simultaneous high spatial and spectral resolution needed to accurately map the relevant SP modes of the plasmonic waveguides at a single nanoparticle level. ${ }^{39}$ For these measurements, the waveguides are deposited on a thin silicon nitride membrane and characterized in a transmission electron microscope (TEM). When the waveguide is excited by a high-energy monochromatic electron beam, some of the electrons are inelastically scattered by the structure, resulting in an energy loss of the electron through the excitation of different SP modes. The excitation of different SP modes depends strongly on the position of the electron beam (Figure 2). The SP modes are analyzed through EELS spectra at selected positions (Figure 2a, d) and by spatially resolved EELS maps at selected resonant energies (Figure 2b, e). 

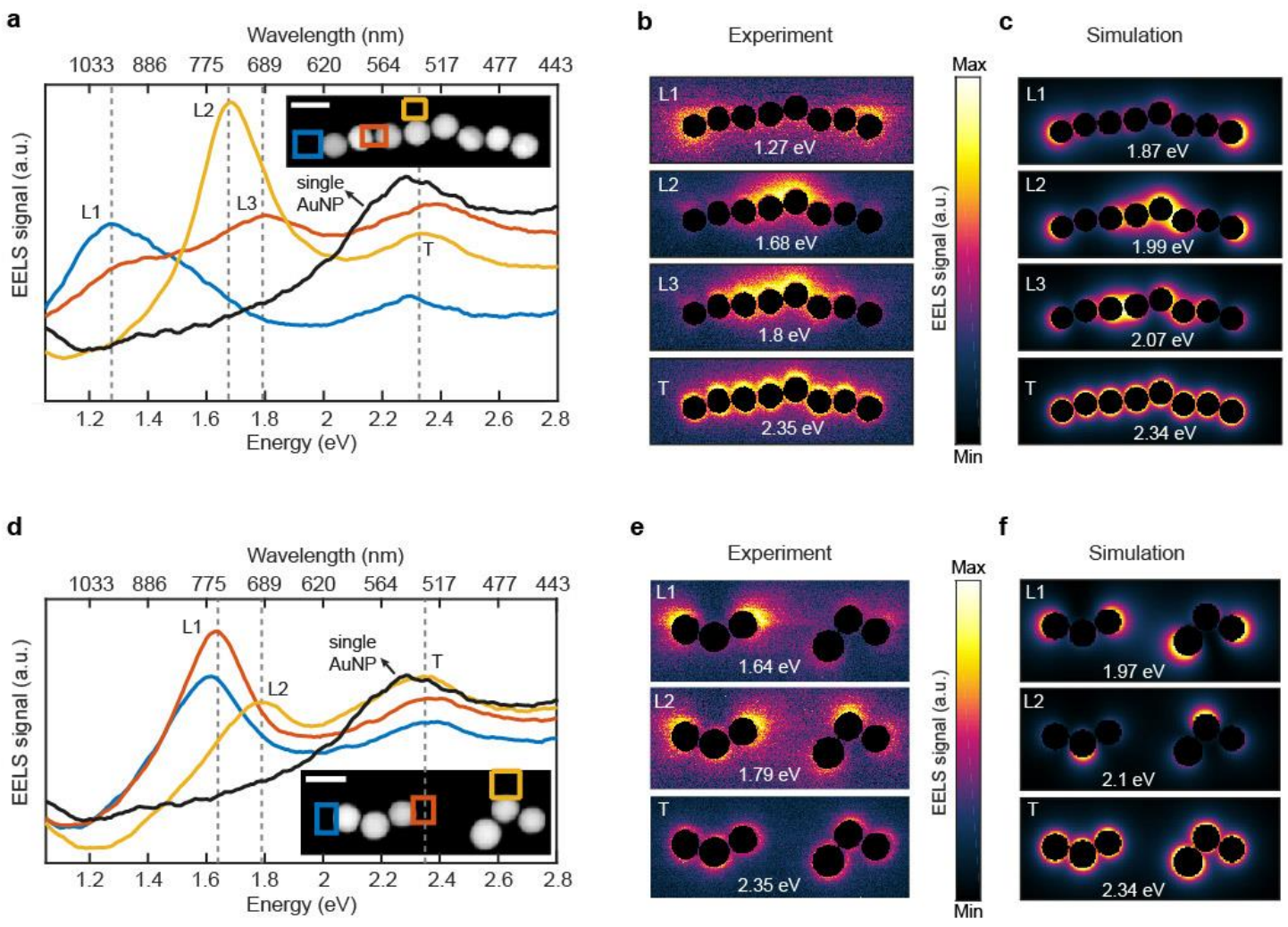

Figure 2. Electron energy loss characterization of complete and incomplete waveguides. (a) Experimental EELS spectra of complete waveguide acquired within the squares in corresponding colors in the STEM micrographs insets. $(b, c)$ Experimental and simulated EELS maps at different energies. (d-f) Same as (a-c) but for the incomplete waveguide. Propagating SP modes labelled according to their mode number (L: 1, 2, 3) from lowest to highest energy and longitudinal (L) / transverse (T) excitation in respect to the geometric arrangement. Scale bars: $50 \mathrm{~nm}$.

The energy loss peaks for longitudinal (L) modes, where the induced dipoles are parallel to the nanoparticle chain, and the transverse $(\mathrm{T})$ mode, where dipoles are perpendicular to the AuNP chain $^{4}$ (Figure S2), can clearly be distinguished. The energies of the L modes are red-shifted 
while the energy of the $\mathrm{T}$ mode $(2.35 \mathrm{eV}, 527 \mathrm{~nm})$ is slightly blue-shifted compared to single AuNPs $(2.3 \mathrm{eV}, \sim 539 \mathrm{~nm}$, see Figure S3a) due to the strong SP coupling between AuNPs. The lowest energy mode L1, referred to as super-radiant mode, ${ }^{32,40}$ is detected at $1.27 \mathrm{eV}(\sim 976 \mathrm{~nm})$. The next $\mathrm{L}$ modes, $\mathrm{L} 2$ and $\mathrm{L} 3$, are detected at $1.68 \mathrm{eV}(\sim 738 \mathrm{~nm})$ and $1.8 \mathrm{eV}(\sim 689 \mathrm{~nm})$, respectively. $\mathrm{L}$ modes with even numbers (L: $2,4, \ldots)$ are so-called dark modes or non-radiative modes as they do not have a net dipole moment, whereas bright modes $(\mathrm{L}: 1,3, \ldots)$ have a net dipole moment. ${ }^{40}$ Dark modes are also considered to effectively support propagation of SP modes along the closely spaced metal nanoparticle due to lower radiative losses than bright modes. $^{32}$

In Figure 2, we compare experimental EELS results ( $a, b, d$, and e) with boundary element method (BEM) simulations (c, f) that are carried out on the same particle arrangement as in the experiment (see Methods). The simulated modes are in good agreement with the experimental results. The slight energy differences between experimental and simulation results (Figure 2) can be attributed to simplifications required for the simulations, i.e., neglecting DNA capping on the AuNPs surface, assumption of perfect spheres, and compensating for the silicon nitride substrate by an effective media. The occurrence of different modes resembles that from stochastically assembly AuNP chains ${ }^{40}$ or that of multilayer nanoparticle ribbons. ${ }^{11,12}$ The incomplete waveguide reveals the plasmon resonances of individual trimers whereas the complete waveguides support continuous SP propagation along the entire chain of eight particles (Supporting Table S1). 

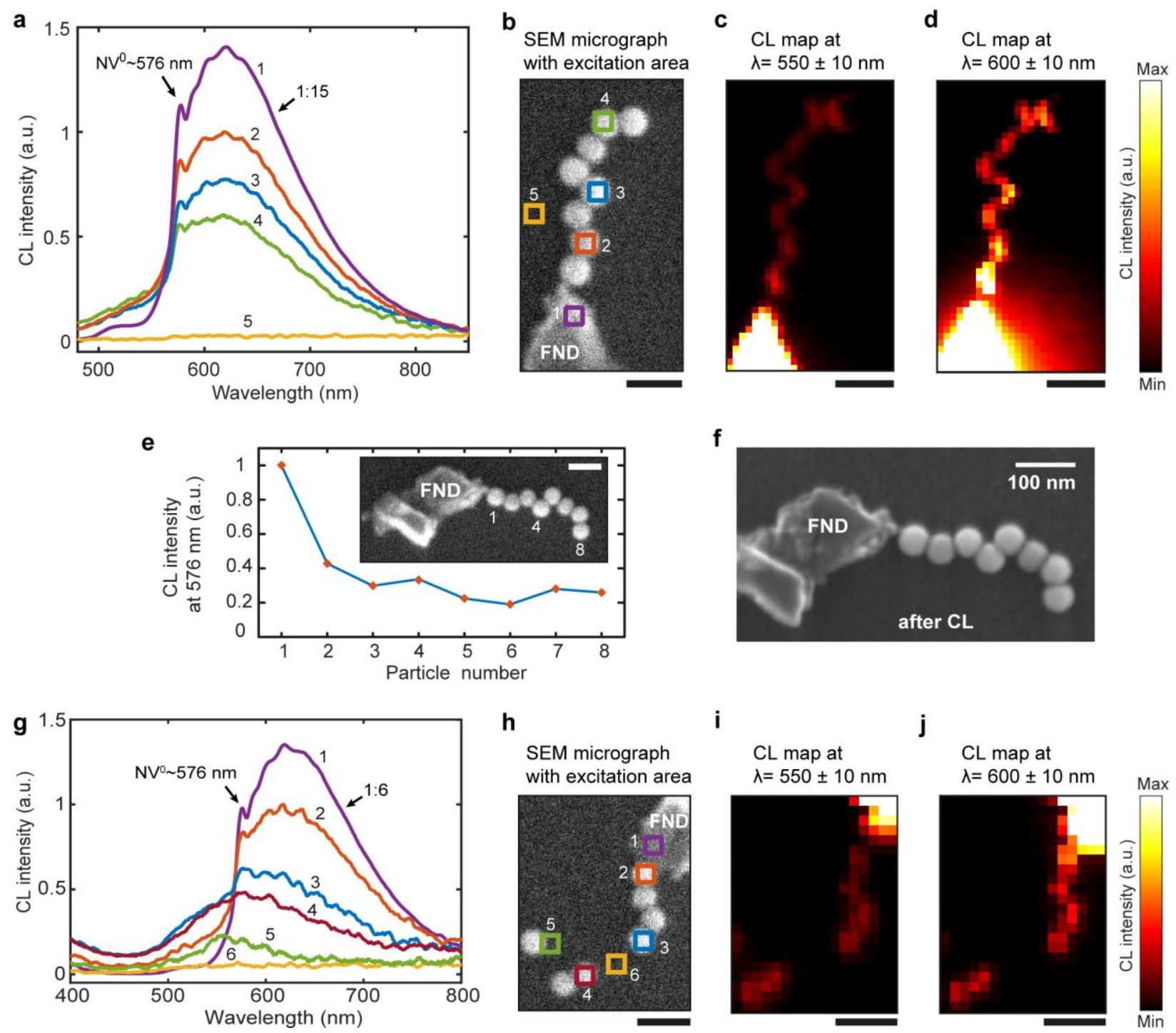

Figure 3. Energy transfer through plasmonic waveguides. Cathodoluminescence (CL) spectra when illuminating waveguides adjacent to a fluorescent nanodiamond (FND). The CL spectra of the complete (a) and incomplete (g) waveguides taken at electron beam excitation areas indicated by squares in the corresponding colors in the SEM micrographs $(b, h)$. The intensity of the FND emission under direct e-beam illumination (purple) is reduced 15-fold (a) or 6-fold (g) for clarity. Spatially resolved CL maps at the detection wavelengths of $550 \mathrm{~nm}( \pm 10 \mathrm{~nm})(\mathrm{c}$, i) and $600 \mathrm{~nm}$ $( \pm 10 \mathrm{~nm})(\mathrm{d}, \mathrm{j})$, showing CL intensity as a function of electron beam excitation positions. (e) CL intensity at the wavelength of $576 \mathrm{~nm}$ (corresponding to $\mathrm{NV}^{0}$ ) as a function of excitation position 
in the complete waveguide. (f) A high-resolution SEM micrograph of the same waveguide with a FND, taken after the CL measurements. Scale bars: $100 \mathrm{~nm}$.

Next, we demonstrate low-loss SP propagation through our nanoparticle waveguides towards a FND and detect it via FND light emission, thus realizing nanoscale frequency conversion. The FNDs are extremely photostable, bright, relatively insensitive to the chemical and physical environments and therefore ideal for electron microscopy studies ${ }^{37,41}$ (see photoluminescence and CL spectra in Figure S4). Moreover, they are promising nanostructures for single photon quantum information technology. ${ }^{37}$ In our experiments, we use commercial FNDs that unfortunately cannot directly attach to the DNA nanostructures. Our focus is, however, to demonstrate the strongly reduced losses and subwavelength field confinements, and for this, the lower assembly yield by the random placement of FNDs is tolerated. For future applications, quantum dots ${ }^{42}$ or fluorophores, which have better defined shapes, size distributions, and contact interfaces with the AuNPs, can be site-specifically attached to the waveguides.

Figure 3 shows the CL measurements on isolated complete and incomplete waveguides next to a single FND (for CL set-up, see Figure S5). FNDs feature a nitrogen-vacancy (NV) point defect in the diamond lattice, where nitrogen replaces a carbon atom next to a vacancy, producing characteristic zero-phonon lines (ZPLs) at $576 \mathrm{~nm}$ for the neutral charge state (NV0) and $637 \mathrm{~nm}$ for the negative charge state (NV-). When the electron beam is positioned on the FND, an intense FND-characteristic CL spectrum featuring the $\mathrm{NV}^{0}$ emission is observed. In the complete waveguide, this characteristic FND spectrum is also observed when exciting any of the AuNPs of the waveguide, including the particle furthest from the FND, which is approximately $350 \mathrm{~nm}$ away from the FND (Figure 3a). These CL spectra are markedly different in the absence of the 
FND (see Figure S6 and for simulation results, see Figure S7). This provides strong evidence that the electron-beam-excited SP modes in the particle chain waveguide propagate energy to the FND, which then emits light to the far field. Propagation losses can be estimated from the drop in CL intensity at the emission wavelengths of the FND (Figure 3d at $600 \mathrm{~nm}$ and Figure $3 \mathrm{e}$ at $576 \mathrm{~nm}$ ). The highest intensity decrease occurs between particle 1 and 2 (the ones closest to the FND). However, CL intensities only drop by a few percent between particles 2 and 8 indicating an efficient waveguiding over several hundred nanometers. The intensity decay between particles 1 and 8 implies a propagation loss of $5.9 \mathrm{~dB}$ for the entire waveguide $(\sim 350 \mathrm{~nm})$, which corresponds to $0.8 \mathrm{~dB}$ per $50 \mathrm{~nm}$. A high-resolution SEM image of the waveguide after the $\mathrm{CL}$ measurements revealed that the waveguide remains intact and that no fusing of the AuNPs occurs (Figure $3 \mathrm{f})$.

To provide further evidence that indeed energy propagation through the AuNPs is responsible for the emission of the FND, we analyzed an incomplete waveguide as a negative control (Figure 3h). As expected, the particle closest to the FND excites the FND and exhibits a clear ZPL peak. Excitation of the particle before the gap (Figure 3g-h, spectrum 3) still shows a ZPL peak but the spectrum is broader and blue-shifted compared to the FND emission and is likely a combination of the $\mathrm{T}$ mode of the tetramer and the FND emission. Moreover, the observed waveguide performance of the tetramer is lower than that of the octamer waveguide (see Figure 3), which can be explained by a lower coupling efficiency of the L3 and L4 mode to the $\mathrm{NV}^{0}$ centers. The ZPL peak cannot be identified anymore in the CL spectrum when exciting the residual AuNP dimer beyond the $\sim 82 \mathrm{~nm}$ gap (Figure $3 \mathrm{~h}$, spectrum 4 ). The broad spectrum shows a maximum at $\sim 570 \mathrm{~nm}$ that is likely to be dominated by one of the longitudinal modes and the $\mathrm{T}$ mode of the dimer. No FND emission can be detected for a single isolated AuNP (Figure 3h, spectrum 5), 
which was most likely detached from the 6-HB during sample preparation or drying. This AuNP is $\sim 230 \mathrm{~nm}$ away from the FND, and its CL spectrum corresponds to that of an isolated particle (Figure S3d). Further control experiments with isolated AuNPs near FNDs (Figure S8) confirm that no coupling to the FND takes place if an AuNP is $\geq \sim 85 \mathrm{~nm}$ away from the FND. However, shorter distances allow coupling to the FND to occur as indicated by a red-shift compared to the CL spectrum of free AuNPs without a FND in their vicinity.

For further insight into the experimentally observed light propagation in the nanoparticle chain waveguides, we perform total-field simulations of our waveguide system, accounting for the silicon substrate and the thin oligonucleotide shell (Figure 4, Supporting note 1). We approximated the electron beam by a dipole source at one end of the waveguide. Early theoretical studies suggested that the $\mathrm{T}$ or $\mathrm{L}$ mode enable waveguiding ${ }^{7,43}$, however, more recently, dark modes were proposed for waveguiding and show reduced radiative losses. ${ }^{32,11,12}$ Our simulations also indicate significant losses for the T mode $(5.3 \mathrm{~dB} / 50 \mathrm{~nm}$ at $562 \mathrm{~nm})$ and the highest energy L mode (L4: $5.3 \mathrm{~dB} / 50 \mathrm{~nm}$ at $518 \mathrm{~nm}$ ) and lower losses for the lower energy L3 mode $(3.1 \mathrm{~dB} / 50 \mathrm{~nm}$ at $680 \mathrm{~nm})$ and the $\mathrm{L} 2$ mode $(4.1 \mathrm{~dB} / 50 \mathrm{~nm}$ at $750 \mathrm{~nm}$; Figure 4 and Table $\mathrm{S} 1)$. Since the excitation wavelength needs to be shorter than the emission of the $\mathrm{NV}^{0}$ center (shorter than $576 \mathrm{~nm}$ ), coupling to the FND should be ineffective for the modes with a lower energy than that of the FND emission. The modes are, however, spectrally broad, and slight increases of the gaps size to 3-5 nm (Figure S9) cause a blue shift of the modes so that they can efficiently couple to the FND. Deviations from the ideal linear geometry has little influence on the mode structure (Figure S10). We have added further discussion to the supporting information to support this hypothesis (see Supporting Note 1). 

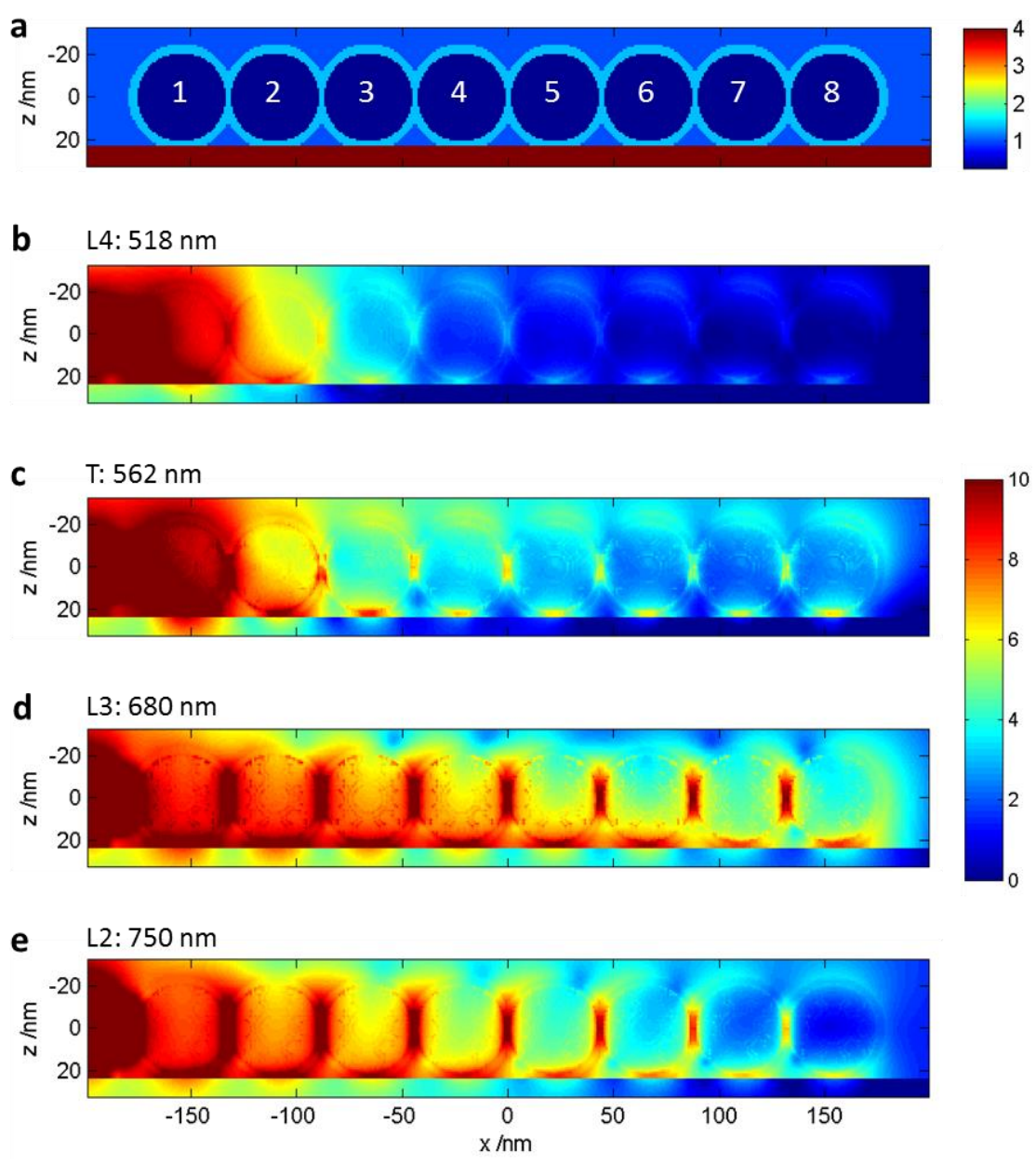

Figure 4. Schematic illustration by refractive index image of the dielectric shelled gold nanoparticles on a silicon substrate in air (a). Integrated electric field distribution for plasmonic waveguide modes at (b) $518 \mathrm{~nm}$ (L4 mode), (c) $562 \mathrm{~nm}$ (T mode), (d) $680 \mathrm{~nm}$ (L3 mode), and (e) $750 \mathrm{~nm}$ (L2 mode). Color bars: logarithmic scale.

In conclusion, we demonstrate gold nanoparticle chain waveguides that can efficiently propagate energy over several hundred nanometers with a loss of only $0.8 \mathrm{~dB}$ per $50 \mathrm{~nm}$, while retaining a deep subwavelength mode size of $62 \pm 3 \mathrm{~nm}(\sim \lambda / 10)$. The low propagation losses are found to be critically dependent on decreasing the interparticle spacing. We achieve this by a 
DNA-origami-based self-assembly approach with highly monodisperse, single crystalline, AuNPs that are of the same size as the distance between binding sites on the DNA. We directly visualize the energy propagation and out-coupling of energy to the far field by CL imaging spectroscopy at a single device level, realizing a nanoscale light source through sub-wavelength energy conversion. With interparticle spacings of only $2 \mathrm{~nm}$, we find that the nanometer spatial resolution of both EELS and CL are critical for understanding the optical properties of our waveguides, and for future engineering of these devices.

Finally, we simulated how variations in the interparticle distance or the deviation from nonideal linear geometries influences the mode structure and propagation length. Electromagnetic modelling shows that the energy transport along the particle chain is much more efficient via lower order longitudinal SP modes (L3 and L2 mode) than via the T or the super-radiant L1 mode as suggested previously. ${ }^{11,12,32}$ The precise and scalable self-assembly method presented herein can potentially be combined with an accurate positioning of DNA origami structures on silicon wafers ${ }^{44}$ to produce complex geometries such as splitters or sharp corners. We envision that this will enable the integration of nanometer-precise plasmonic components with larger and lower loss technologies for high-speed optical data transmission, quantum information technology, as single photon sources and sensing. 


\section{ASSOCIATED CONTENT}

Supporting Information. The following files are available free of charge.

Methods, supporting figures, tables and notes (supporting_information.pdf)

\section{AUTHOR INFORMATION}

\section{Corresponding Author}

*E-mail thorsten-lars.schmidt@tu-dresden.de or concerning simulations to koenig@ipfdd.de

\section{Present Addresses}

$\dagger$ Department of Micro- and Nanotechnology, Technical University of Denmark, DK-2800

Kongens Lyngby, Denmark.

\section{Author Contributions}

T.L.S. and F.N.G. conceived the study. A.M.S. synthesized the AuNPs. F.N.G. functionalized the AuNPs, designed and assembled the waveguides. C.P.T.McP and F.N.G. performed CL measurements and analysis. S.R. performed EELS measurements and analysis. M.M. and T.K. carried out BEM simulations of EELS maps. T.K simulated CL spectra and dipole excitations. D.J.R. and F.N.G. performed PL measurements and analysis. F.N.G. and M.L. imaged the samples by SEM/tSEM. T.L.S. supervised the study. F.N.G, T.K, S.R. and T.L.S. wrote the manuscript, and all authors discussed the results and revised the manuscript. 


\section{Funding Sources}

This work was funded by the DFG through the Center for Advancing Electronics Dresden (cfaed) as well as Seed Grant 043_2615A6 by the DFG Center for Regenerative Therapies

Dresden (CRTD) to T.L.S.; by EPSRC (UK) (A.Z., C.P.T.McP., D.J.R.); the Royal Society and Wolfson Foundation (A.Z); research grant (VKR023371) from VILLUM FONDEN (S.R.); a Multi University Research Initiative (MURIs FA9550-12-1-0488) from the AFOSR (S.R. and M.L.B.); the Volkswagen Foundation through a Freigeist Fellowship to T.K.; and the cfaed through an inspire grant (F.N.G.).

\section{Notes}

The authors declare no competing financial interest.

\section{ACKNOWLEDGMENT}

We thank L. Eng, M. Mertig, A. Hille and A. Krasavin for helpful discussions; S. Gupta and D. P. N. Goncalves for producing artistic waveguides schemes; and the Biomod (biomod.org) team "Dresden DNAmic" (2014) for the help in the early stage of this project.

\section{REFERENCES}

(1) Ozbay, E. science 2006, 311 (5758), 189-193.

(2) Lal, S.; Link, S.; Halas, N. J. Nat. Photonics 2007, 1 (11), 641-648.

(3) Zia, R.; Schuller, J. A.; Chandran, A.; Brongersma, M. L. Mater. Today 2006, 9 (7), 2027.

(4) Brongersma, M. L.; Hartman, J. W.; Atwater, H. A. Phys. Rev. B 2000, 62 (24), R16356.

(5) Maier, S. A. Springer: New York, 2007.

(6) Halas, N. J.; Lal, S.; Chang, W.-S.; Link, S.; Nordlander, P. Chem. Rev. 2011, 111 (6), 3913-3961.

(7) Quinten, M.; Leitner, A.; Krenn, J. R.; Aussenegg, F. R. Opt. Lett. 1998, 23 (17), 1331. 
(8) Maier, S. A.; Kik, P. G.; Atwater, H. A. Appl. Phys. Lett. 2002, 81 (9), 1714.

(9) Maier, S. A.; Kik, P. G.; Atwater, H. A.; Meltzer, S.; Harel, E.; Koel, B. E.; Requicha, A. A. G. Nat. Mater. 2003, 2 (4), 229-232.

(10) Nomura, W.; Ohtsu, M.; Yatsui, T. Appl. Phys. Lett. 2005, 86 (18), 181108.

(11) Solis, D.; Willingham, B.; Nauert, S. L.; Slaughter, L. S.; Olson, J.; Swanglap, P.; Paul, A.; Chang, W.-S.; Link, S. Nano Lett. 2012, 12 (3), 1349-1353.

(12) Solis, D.; Paul, A.; Olson, J.; Slaughter, L. S.; Swanglap, P.; Chang, W.-S.; Link, S. Nano Lett. 2013, 13 (10), 4779-4784.

(13) Mirkin, C. A.; Letsinger, R. L.; Mucic, R. C.; Storhoff, J. J. Nature 1996, 382 (6592), $607-609$.

(14) Alivisatos, A. P.; Johnsson, K. P.; Peng, X.; Wilson, T. E.; Loweth, C. J.; Bruchez, M. P.; Schultz, P. G. Nature 1996, 382 (6592), 609-611.

(15) Jones, M. R.; Seeman, N. C.; Mirkin, C. A. Science 2015, 347 (6224), 1260901.

(16) Chao, J.; Lin, Y.; Liu, H.; Wang, L.; Fan, C. Mater. Today 2015, 18 (6), 326-335.

(17) Acuna, G. P.; Möller, F. M.; Holzmeister, P.; Beater, S.; Lalkens, B.; Tinnefeld, P. Science 2012, 338 (6106), 506-510.

(18) Kuzyk, A.; Schreiber, R.; Fan, Z.; Pardatscher, G.; Roller, E.-M.; Högele, A.; Simmel, F. C.; Govorov, A. O.; Liedl, T. Nature 2012, 483 (7389), 311-314.

(19) Klein, W. P.; Schmidt, C. N.; Rapp, B.; Takabayashi, S.; Knowlton, W. B.; Lee, J.; Yurke, B.; Hughes, W. L.; Graugnard, E.; Kuang, W. Nano Lett. 2013, 13 (8), 3850-3856.

(20) Gür, F. N.; Schwarz, F. W.; Ye, J.; Diez, S.; Schmidt, T. L. ACS Nano 2016, 10 (5), 5374 5382.

(21) Vogele, K.; List, J.; Pardatscher, G.; Holland, N. B.; Simmel, F. C.; Pirzer, T. ACS Nano 2016, 10 (12), 11377-11384.

(22) Roller, E.-M.; Besteiro, L. V.; Pupp, C.; Khorashad, L. K.; Govorov, A. O.; Liedl, T. Nat. Phys. 2017, 13 (8), 761-765.

(23) Heck, C.; Kanehira, Y.; Kneipp, J.; Bald, I. Angew. Chem. Int. Ed. 57 (25), 7444-7447.

(24) Rothemund, P. W. K. Nature 2006, 440 (7082), 297-302.

(25) Douglas, S. M.; Dietz, H.; Liedl, T.; Högberg, B.; Graf, F.; Shih, W. M. Nature 2009, 459 (7245), 414-418.

(26) Kuzyk, A.; Schreiber, R.; Zhang, H.; Govorov, A. O.; Liedl, T.; Liu, N. Nat. Mater. 2014, $13(9), 862-866$.

(27) Maier, S.; Kik, P.; Atwater, H. Phys. Rev. B 2003, 67 (20).

(28) Coenen, T.; Vesseur, E. J. R.; Polman, A.; Koenderink, A. F. Nano Lett. 2011, 11 (9), 3779-3784.

(29) Barnes, W. L.; Dereux, A.; Ebbesen, T. W. Nature 2003, 424 (6950), 824-830.

(30) Oulton, R. F.; Sorger, V. J.; Genov, D. A.; Pile, D. F. P.; Zhang, X. Nat. Photonics 2008, 2 (8), 496-500.

(31) Lamprecht, B.; Krenn, J. R.; Schider, G.; Ditlbacher, H.; Salerno, M.; Felidj, N.; Leitner, A.; Aussenegg, F. R.; Weeber, J. C. Appl. Phys. Lett. 2001, 79 (1), 51-53.

(32) Willingham, B.; Link, S. Opt. Express 2011, 19 (7), 6450-6461.

(33) Hanske, C.; Tebbe, M.; Kuttner, C.; Bieber, V.; Tsukruk, V. V.; Chanana, M.; König, T. A. F.; Fery, A. Nano Lett. 2014, 14 (12), 6863-6871.

(34) Agarwal, N. P.; Matthies, M.; Gür, F. N.; Osada, K.; Schmidt, T. L. Angew. Chem. Int. Ed. 2017, 56 (20), 5460-5464.

(35) Colliex, C.; Kociak, M.; Stéphan, O. Ultramicroscopy 2016, 162, A1-A24. 
(36) Koh, A. L.; Bao, K.; Khan, I.; Smith, W. E.; Kothleitner, G.; Nordlander, P.; Maier, S. A.; McComb, D. W. ACS Nano 2009, 3 (10), 3015-3022.

(37) Aharonovich, I.; Greentree, A. D.; Prawer, S. Nat. Photonics 2011, 5 (7), 397-405.

(38) Kuttge, M.; Vesseur, E. J. R.; Koenderink, A. F.; Lezec, H. J.; Atwater, H. A.; García de Abajo, F. J.; Polman, A. Phys. Rev. B 2009, 79 (11), 113405.

(39) García de Abajo, F. J. Rev. Mod. Phys. 2010, 82 (1), 209-275.

(40) Barrow, S. J.; Rossouw, D.; Funston, A. M.; Botton, G. A.; Mulvaney, P. Nano Lett. 2014, 14 (7), 3799-3808.

(41) Schietinger, S.; Barth, M.; Aichele, T.; Benson, O. Nano Lett. 2009, 9 (4), 1694-1698.

(42) Weichelt, R.; Leubner, S.; Henning-Knechtel, A.; Mertig, M.; Gaponik, N.; Schmidt, T.L.; Eychmüller, A. Small 2016, 12 (34), 4763-4771.

(43) Maier, S. A.; Brongersma, M. L.; Kik, P. G.; Meltzer, S.; Requicha, A. A. G.; Atwater, H. A. Adv. Mater. 2001, 13 (19), 1501-1505.

(44) Gopinath, A.; Miyazono, E.; Faraon, A.; Rothemund, P. W. K. Nature 2016, 535 (7612), 401-405. 